\title{
Influence of humic compounds on bioluminescence of immobilized photobacteria
}

Senko O. ${ }^{1,2}$, Stepanov N. ${ }^{1,2}$, Efremenko E. ${ }^{1,2}$

${ }_{1}^{1}$ Lomonosov Moscow State University, Moscow, Russia, elena_efremenko@list.ru

${ }^{2}$ Emanuel Institute of Biochemical Physics, RAS, Moscow, Russia

doi: 10.36291/HIT.2019.senko.077

An analytical test based on the use of bioluminescent bacteria is characterized by a quick response and qualitative analysis. Additionally, it is possible to adapt existing portable devices for its use [1,2]. Particular attention is paid to the use of biosensitive elements in the form of immobilized bioluminescent bacterial cells $[3,4]$. It was previously shown for a biosensitive element developed on the basis of the bacterial Photobacterium phosphoreum cells immobilized in macroporous poly(vinyl alcohol) cryogel (PVA cryogel). It was found that such a biosensitive element can be successfully used for analyzing the toxicity of media containing heavy metal ions, phenol derivatives, and organophosphorous pesticides, both in a discrete and flow-through mode [3,4]. The PVA cryogel as a carrier positively influences the stability of the cell bioluminescent signal in the absence of toxins and does not affect the bioluminescent registration with the modern devices used in the experiments.

The use of immobilized bacterial cells of $P$. phosphoreum for primary screening of humic compounds (HCs), proposed for use to suppress gas evolution from landfills, from the point of view of their toxic effect on cells of microorganism, was proposed in this work.

It was shown that in the studied range of concentrations of HCs $(0.1-10 \mathrm{~g} / \mathrm{I})$ there is a notable direct correlation between the concentration of $\mathrm{HCs}$ and the residual luminescence intensity of immobilized $P$. phosphoreum cells. Moreover, those HCs that had the maximum effect in the case of suppression of the methanogenesis process [5], maximally inhibited the luminescence of photobacteria

Thus, it can be argued that the biosensitive element in the form of immobilized $P$. phosphoreum cells can be used a primary screening tool for assessing the effect of HCs on cells of microorganisms.

Acknowledgements. This research was funded by the Russian Foundation for Basic Research (Grant No. 18-29-25065).

References

1. Michelini E. et al. // Anal. Bioanal. Chem. 2013. 405:6155-6163.

2. Roggo C. et al. // Curr. Opin. Biotechnol. 2017. 45:24-33.

3. Efremenko E.N. et al. // Luminescence. 2016. 31:1283-1289.

4. Efremenko E.N. et al. // Appl. Biochem. Microbiol. 2014. 50:477-482.

5. Stepanov N. et al. // Sustainability. 2019. 11:3158-11p. 